\title{
Detección de parásitos gastrointestinales en hurones (Mustela putorius furo) mediante análisis coprológico
}

\section{Detection of gastrointestinal parasites in ferrets (Mustela putorius furo) by coprological analysis}

\author{
I. Troncoso ${ }^{1,6}$, C. Fischer ${ }^{2}$, S. Valenzuela ${ }^{2}$, I. Fernández ${ }^{3}$, A. Robles ${ }^{2}$, \\ F. Villalobos ${ }^{4}$, D. González-Acuña ${ }^{5}$
}

\section{Resumen}

\begin{abstract}
El objetivo del estudio fue determinar la presencia de parásitos gastrointestinales zoonóticos mediante examen coproparasitológico en hurones (Mustela putorius furo) criados como mascotas y el rol de las variables sexo y edad. Se analizaron las heces de 30 hurones, 16 [53.3\%] de tiendas de mascotas y 14 [46.7\%] de hogares de la ciudad de Santiago y Concepción, Chile. Para el diagnóstico se utilizó la técnica coproparasitaria de Burrows, que permite detectar huevos de helmintos, quistes y trofozoítos de protozoos. Tres muestras (10\%) resultaron positivas a protozoos, una a Entamoeba coli, en un macho de 6 meses de una tienda de mascotas, y dos a Endolimax nana en una hembra de 3 meses y un macho de 2 años, ambos de hogares. Este sería el primer registro para las dos especies de protozoos comensales en hurones en Chile, los que probablemente fueron adquiridos por el estrecho contacto de los mustélidos con los humanos.
\end{abstract}

Palabras clave: prevalencia; hurones; endoparásitos; heces; coproparasitario

${ }^{1}$ Escuela de Medicina Veterinaria, Facultad de Medicina Veterinaria y Agronomía, Universidad de las Américas, Concepción, Chile

${ }^{2}$ Escuela de Medicina Veterinaria, Facultad de Recursos Naturales y Medicina Veterinaria, Universidad Santo Tomás, Concepción, Chile

${ }^{3}$ Departamento de Parasitología, Facultad de Ciencias Biológicas, Universidad de Concepción, Chile

${ }^{4}$ Escuela de Medicina Veterinaria, Facultad de Recursos Naturales y Medicina Veterinaria, Universidad Santo Tomás, Talca, Chile

${ }^{5}$ Facultad de Ciencias Veterinarias, Universidad de Concepción, Chillán, Chile

${ }^{6}$ E-mail: ignacio.troncoso@edu.udla.cl

Recibido: 22 de octubre de 2018

Aceptado para publicación: 15 de mayo de 2019 
The aim of this study was to determine the presence of zoonotic gastrointestinal parasites by coprological examination in ferrets (Mustela putorius furo) reared as pets and the role of sex and age. The faeces of 30 ferrets were analysed, 16 [53.3\%] from pet stores and 14 [46.7\%] from homes in the city of Santiago and Concepción, Chile. Burrows' coprological technique was used for the diagnosis, which allows detecting eggs of protozoan helminths, cysts and trophozoites Three samples $(10 \%)$ tested positive for protozoa, one for Entamoeba coli, in a 6 month old male from a pet store, and two for Endolimax nana in a 3-month-old female and a 2-year-old male from private homes. This would be the first record for the two species of protozoa commensals in ferrets in Chile, which were probably acquired by the close contact of mustelids with humans.

Key words: prevalence; ferrets; endoparasites; feces; coprological

\section{INTRODUCCIÓN}

En el mundo en general, la tenencia de animales silvestres o exóticos se ha incrementado ya que muchas personas se inclinan por adquirir mascotas no tradicionales, las cuales presentan características novedosas en comparación a los animales considerados domésticos, ya sea por sus características biológicas, fenotípicas o cuidados en general (Oroza, 2008). Según Abarca et al. (2002), la tenencia de mascotas exóticas en Chile se ha venido incrementando. Los registros indican la presencia de mascotas en $58 \%$ de los hogares de sujetos inmunocomprometidos (IC), tanto adultos como niños, siendo en el 5\% para mascotas exóticas (Abarca et al., 2002). Posteriormente, una encuesta realizada a 11060 viviendas de Santiago de Chile reveló que el $85.9 \%$ poseía una mascota en su hogar, y con una proyección de población estimada de 146 hurones para el año 2002 (Ibarra et al., 2003). El ultimo antecedente, fue realizado por Wuth (2012), quien analizó la venta de especies exóticas en tiendas de mascotas del Gran Santiago, donde el caso del hurón fue equivalente al $0.8 \%(20 / 2471)$.
El hurón (Mustela putorius furo) puede presentar diversas patologías (Meredith y Redrobe, 2007), siendo el parasitismo gastrointestinal poco común (Aguilar et al., 2010), por lo que estas mascotas no se desparasitan rutinariamente a menos que existan signos y pruebas de laboratorio que sustenten la necesidad de una profilaxis antiparasitaria (Vinke et al., 2012). Pueden infectarse con parásitos helmintos de caninos y felinos como Toxocara spp, Toxascaris leonina, Ancylostoma spp y Dipylidium caninum (Powers, 2009). Estas infecciones son poco comunes, ya que los parásitos pueden estar en números más bajos que en felinos y caninos, lo cual puede deberse a una exposición reducida, rápido tránsito intestinal, ausencia de transmisión transplacentaria/ transmamaria o la combinación de estos factores (Wright, 2012).

En Chile, no se han realizado investigaciones sobre los parásitos gastrointestinales de hurones, por lo que, este estudio tuvo por objetivo determinar la presencia de parásitos gastrointestinales, evaluar el potencial zoonótico de los parásitos aislados y determinar si existe asociación con las variables sexo y edad. 


\section{Materiales y Métodoos}

En agosto de 2015 se realizó un muestreo no probabilístico de tipo dirigido a 30 hurones en las ciudades de Santiago (S) y Concepción (C), Chile. De estos, 16 (53.3\%) pertenecían a tiendas de mascotas (12 S y 4 C) y 14 fueron muestreadas en hogares (46.7\%) (10 S y 4 C). La información de los animales fue obtenida de registros de pacientes de una veterinaria dedicada a la atención de animales exóticos (en C) y de una cadena de tiendas dedicados a la venta de especies exóticas (en S).

Solo se consideraron hurones que no habían recibido medicación contra parásitos internos en al menos un mes previo a la toma de la muestra (Miller et al., 2006), sin discriminar por la cantidad de animales por hogar o tienda. En todos los casos, se contó con la firma previa de la carta de consentimiento y confidencialidad de los resultados por parte del propietario del animal o dueño de la tienda.

La recolección de las deposiciones fue efectuada por miembros del grupo de investigación in situ y se realizó durante las primeras horas de la mañana, ya que es el momento en que se registra una mayor liberación de huevos o larvas (Garrido et al., 2011). Se recolectó no menos de $3 \mathrm{~g}$ de material fecal por animal, inmediatamente después de emitidas. Las muestras fueron colocadas en frascos plásticos conteniendo $10 \mathrm{ml}$ de preservante PAF como fijador (López et al., 2006), almacenados en un cooler a $4{ }^{\circ} \mathrm{C}$ y transportados al Laboratorio de Medicina Veterinaria de la Universidad Santo Tomás, sede Concepción para su análisis.

Se utilizó la técnica de Burrows (1967) como método diagnóstico, puesto que permite determinar huevos de helmintos, quistes y trofozoítos de protozoos. El sedimento fue observado en un microscopio óptico (Olympus CX31) a 10x y 40x (López et al., 2006; Garrido et al., 2011). La sensibilidad del examen de una única muestra en fresco de heces es de $35-50 \%$, la cual podría haberse incrementado al $70 \%$ si se considerase un muestreo seriado (Chajmi, 2007). La identificación de los parásitos encontrados se confirmó de acuerdo con su morfología (Blanco y Galiano, 1989; Jeffrey y Leach, 1991) y por comparación con preparaciones de microteca de parásitos encontrados comúnmente en perros y gatos, utilizados como apoyo y material de referencia.

El estudio fue de tipo descriptivo y transversal, se determinó el porcentaje de hospederos parasitados y la riqueza parasitaria (número de taxones parasitarios por hospedador parasitado) (Pardo et al., 2004). Se utilizó la prueba no paramétrica de Fisher para evaluar posibles diferencias entre individuos parasitados y las variables cualitativas de sexo y edad.

\section{Resultados y Discusión}

El 10\% ( $\mathrm{n}=3)$ de los hurones resultaron positivos para endoparásitos intestinales. Se encontraron quistes de dos especies de protozooarios: Endolimax nana y Entamoeba coli. La muestra positiva a Entamoeba coli fue colectada en un macho de 6 meses, proveniente de una tienda de mascota, mientras que las dos muestras positivas a Endolimax nana fueron de una hembra de tres meses y un macho de dos años, ambos de hogares. No hubo diferencia significativa para las variables analizadas.

Este es el primer estudio de parasitismo en hurones realizado en Chile. Como antecedente, destaca el estudio de d'Ovidio et al (2014) en Italia con 50 hurones, donde el $28.0 \%(14 / 50)$ presentaba huevos de Ancylostoma y el 2\% (1/50) ooquistes de Sarcocystis, mediante la técnica de pellets FLOTAC para la detección y recuento de helmintos, huevos/larvas y quistes/ooquistes de protozoarios. En Latinoamérica destaca el estudio de Da Rosa et al. (2010) en el sur 
de Brasil con dos hurones de 3 años, utilizando el método de frotis directo, detectando oocistos de Cryptosporidum sp en las heces de ambos animales. A pesar del uso de diferentes técnicas coprológicas, existen estudios donde se demuestra que no hay diferencias entre las técnicas de flotación para el diagnóstico de protozoos (de Lozano, 1982) ni para los geohelmintos (Restrepo et al., 2013).

Cryptosporidium sp en hurones ha sido reportado en otros países. Por ejemplo, Rehg et al. (1988) encontraron $40 \%$ de individuos positivos para este coccidio en hurones jóvenes de EEUU. Así mismo, se han reportado casos fatales en hurones mantenidos en cautiverio (Gómez Villamandos et al., 1995). Además, Abe et al. (2008) en Japón y Sledge et al. (2011) en EEUU encontraron protozoos de los géneros Eimeria y Cryptosporidium empleando técnicas moleculares como análisis de secuenciación del gen ribonucleico ribosómico de la subunidad pequeña de ácido (SSU-rDNA) y PCR desde tejido intestinal.

No existen antecedentes que demuestren la presencia de Entamoeba coli y Endolimax nana en hurones; sin embargo, López et al. (2006) detectaron la presencia de estos protozoos en Entamoeba coli en $33.1 \%$ de los perros y $31.7 \%$ de los gatos y Endomilax nana en el $34.3 \%$ de los perros $(n=972)$ y $32.2 \%$ de los gatos $(n=230)$ con signología digestiva en Santiago de Chile. Los protozoos hallados en este estudio son consideradas no patógenos para el ser humano (Sard et al., 2011), quien actúa como reservorio natural; mientras que los perros y gatos serían hospedadores accidentales y constituirían un ejemplo de antropozoonosis (Meloni et al., 1995).

La prevalencia mundial de Entamoeba coli en humanos aparentemente sanos oscila entre 10 y 40\% (Sard et al., 2011). En el caso de Endolimax nana se trata de una de las amebas intestinales más pequeñas que colonizan al ser humano (Sard et al., 2011), de distribución cosmopolita y con prevalencia similar a Entamoeba coli. Ambas son de transmisión fecal-oral y residen en el intestino grueso del humano, donde los quistes y trofozoítos de estas especies pasan a las heces. Los quistes se encuentran típicamente en las heces formadas, mientras que los trofozoítos se encuentran en heces diarreicas (Apt, 2014).

La información de amebas intestinales no patógenas humanas en hurones no ha sido descrita hasta la fecha; sin embargo, existen algunos estudios que pudiesen explicar la presencia de estos parásitos en el hurón. Existe la posibilidad que el hurón adquiera estas amebas al cohabitar con animales domésticos como perros o gatos (Plaut et al., 1996; Halsby et al., 2014); sin embargo, d'Ovidio et al. (2014) demuestran que la interacción entre hurones y otras mascotas es extremadamente rara, donde los hurones de propiedad privada que resultaron positivos compartían su ambiente con perros o gatos fueron todos negativos. Se presume que la infección de hurones con estos protozoos podría provenir de diferentes fuentes, desde propietarios portadores de los protozoos asociados a deficientes condiciones higiénicas de alimentación y agua de bebida, la probable intervención de un vector como Musca domestica para la infestación parasitaria (Romero et al., 2004) o por posible contacto con especies domésticas como caninos o felinos, generando una infección espuria en los hurones (Plaut et al., 1996; Halsby et al., 2014).

Las variables sexo y edad no fueron determinantes como un factor asociado a la presencia de los protozoos. Según Madrid et al. (2012), el sexo no es un factor de riesgo para el parasitismo en hurones, pero si la edad, ya que se ha determinado mayor parasitismo por algunas especies de protozoos en individuos juveniles, hecho que no se comprobó en este estudio. 


\section{Literatura Citada}

1. Abarca K, Dabanch J, Jofré L. 2002. Tenencia de mascotas en población sana e inmunodeprimida de Santiago. En: XIX Congreso Chileno de Infectología. Santiago de Chile.

2. Abe N, Tanoue T, Ohta G, Iseki M. 2008. First record of Eimeria furonis infection in a ferret, Japan, with notes on the usefulness of partial small subunit ribosomal RNA gene sequencing analysis for discriminating among Eimeria species. Parasitol Res 103: 967-970. doi: 10.1007/s00436-008-1037-x

3. Aguilar RF, Hernández SM, Divers SJ, Perpiñán D. 2010. Atlas de medicina de animales exóticos. Argentina: Intermédica. $496 \mathrm{p}$.

4. Apt W. 2014. Comensales tubo digestivo. En: Parasitología humana. Learning Center. p 268-269.

5. Blanco J, Galiano J. 1989. Atlas de coprología, digestión y parásitos. España: Asociación Española de Farmacéuticos Analistas. 200 p.

6. Burrows RB. 1967. A new fixative and techniques for the diagnosis of intestinal parasites. Am J Clin Pathol 48: 342-346.

7. Chajmi J. 2007. Determinación de parásitos presentes en una giardiasis y estudio comparativo de los métodos de Ritchie y de Bailenger, en muestras de menores de 12 años, atendidos en el Hospital del Niño de la ciudad de La Paz entre los meses de septiembre de 2005 a febrero de 2006. Tesis de Bioquímico. Bolivia: Univ. Mayor de San Andrés. 84 p.

8. Da Rosa Fanfa V, Farret MH, Da Silva AS, Monteiro SG. 2010. Cryptosporidium spp em furão (Mustela putorius furo) no sul do Brasil. Biotemas 23: 225-227.

9. De Lozano GC. 1982. Estudio comparativo de tres técnicas coproparasitológicas empleadas en el diagnóstico de la giardiasis e hymenolepiasis. Kasmera 10: 1-4.

10. D'Ovidio D, Pepe P, Ianniello D, Noviello E, Quinton JF, Cringoli G, Rinaldi L. 2014. First survey of endoparasites in pet ferrets in Italy. Vet Parasitol 203: 227-230. doi: 10.1016/ j.vetpar.2014.03.019

11. Garrido N, Gallardo P, Ríos P. 2011. Instructivo de trabajo $\mathrm{N}^{\circ} 7$, parasitológico seriado de deposiciones. Método de Burrows. Ministerio de Salud; Chile. p 9-11.

12. Gomez-Villamandos JC, Carrasco L, Mozos E, Hervas J. 1995. Fatal cryptosporidiosis in ferrets (Mustela putorius furo): a morphopathologic study. J Zoo Wildlife Med 26: 539-544.

13. Halsby KD, Walsh AL, Campbell C, Hewitt K, Morgan D. 2014. Healthy animals, healthy people: zoonosis risk from animal contact in pet shops, a systematic review of the literature. PLoS One 9(2): e89309. doi: 10.1371/journal.pone.0089309

14. Ibarra L, Morales MA, Orella P. 2003. Existencias de especies animales de compañía en la ciudad de Santiago, Chile. Avances Cienc Vet 18. doi: 10.5354/ 0719-5273.2010.9172

15. Jeffrey MC, Leach RM. 1991. Atlas of medical helminthology and protozoology, Edinburgh: Longman. $121 \mathrm{p}$.

16. López J, Abarca K, Paredes P, Inzunza E. 2006. Parásitos intestinales en caninos y felinos con cuadros digestivos en Santiago, Chile: consideraciones en Salud Pública. Rev Med Chile 134: 193200. doi: 10.4067/S0034-98872006000200009

17. Madrid Valdebenito V, Torrejon Godoy E, Fernández Fonseca I. 2012. Manual de parasitología humana. Chile: Univ. de Concepción. $216 \mathrm{p}$.

18. Meloni BP, Lymbery AJ, Thompson RC. 1995. Genetic characterization of isolates of Giardia duodenalis by enzyme electrophoresis: implications for reproductive biology, population structure, taxonomy, and epidemiology. $\mathrm{J}$ Parasitol 81: 368-383. doi: 10.2307/ 3283818

19. Meredith A, Redrobe S. 2007. Manual de animales exóticos. British Small Animal Veterinary Association. 446 p. 
20. Miller DS, Eagle RP, Zabel S, Rosychuk R, Campbell TW. 2006. Efficacy and safety of selamectin in the treatment of Otodectes cynotis infestation in domestic ferrets. Vet Rec 159: 748. doi: $10.1136 / v r .159 .22 .748$

21. Oroza P. 2008. Tenencia de animales no convencionales como mascotas entre estudiantes de la Facultad de Veterinaria y Zootecnia de la Universidad Cayetano Heredia. Tesis de Médico Veterinario Zootecnista. Lima, Perú. Univ. Peruana Cayetano Heredia. 19 p.

22. Pardo MC, Garcías F, GeorgeNascimento MG. 2004. La dieta y fauna de endoparásitos del pejesapo Gobiesox marmoratus. Jenyns, 1842 (Pisces: Gobiesocidae) en el litoral central de Chile están conectadas pero no correlacionadas. Rev Chil Hist Nat 77: 627-637. doi: 10.4067/S0716-078X2004000400006

23. Plaut M, Zimmerman EM, Goldstein $\boldsymbol{R} A$. 1996. Health hazards to humans associated with domesticated pets. Annu Rev Public Health 17: 221-245. doi: 10.1146/annurev.pu.17.050196.001253

24. Powers LV. 2009. Bacterial and parasitic diseases of ferrets. Vet Clin North Am Exot Anim Pract 12: 531-561. doi: 10.1016/j.cvex.2009.06.001

25. Rehg JE, Gigliotti F, Stokes DC. 1988. Cryptosporidiosis in ferrets. Lab Anim Sci 38: 155-158.

26. Restrepo IC, Mazo LP, Salazar ML, Montoya MN, Botero JH. 2013. Evaluation of three coproparasitoscopic techniques for the diagnosis of intestinal geohelminthiases. Iatreia 26: 15-24.

27. Romero LC, Tay J, Vega JS, Sánchez DR. 2004. Los artrópodos y su importancia en medicina humana. Rev Fac Med UNAM 47: 192-199.

28. Sard B, Navarro R, Sanchis JG. 2011. Amebas intestinales no patógenas: una visión clinicoanalítica. Enferm Infec Micr Cl 29: 20-28. doi: 10.1016/S0213005X(11)70023-4

29. Sledge DG, Bolin SR, Lim A, Kaloustian LL, Heller RL, Carmona FM, Kiupel M. 2011. Outbreaks of severe enteric disease associated with Eimeria furonis infection in ferrets (Mustela putorius furo) of 3 densely populated groups. J Am Vet Med Assoc 239: 1584-1588. doi: 10.2460/ javma.239.12.1584

30. Vinke CM, Schoemaker NJ. 2012. The welfare of ferrets (Mustela putorius furo $\mathrm{T}$ ): a review on the housing and management of pet ferrets. Appl Anim Behav Sci 139: 155-168. doi: 10.1016/ j.applanim.2012.03.016

31. Wright I. 2012. Parasites of ferrets: sharing with their canine and feline friends. Companion Animal 17: 45-49. doi: 10.1111/j.2044-3862.2012.00145.x

32. Wuth G 2012. Descripción del mercado de pequeños mamíferos exóticos y del ejercicio profesional clínico asociado a éste. Tesis de Médico Veterinario. Chile: Univ. de Chile. 26 p. 